\title{
Ultrasound of the Knee During Voluntary Quadriceps Contraction: A Technique for Detecting Otherwise Occult Effusions
}

\author{
ROBERT W. IKE, ${ }^{1}$ EMILY C. SOMERS, ${ }^{1}$ ERIN L. ARNOLD, ${ }^{2}$ AND WILLIAM J. ARNOLD ${ }^{2}$
}

Objective. To describe 1) a technique that can detect synovial effusions not seen on static ultrasound (US) examination and 2) the characteristics of patients with knee osteoarthritis (OA) for whom this technique proved useful.

Methods. From reviewed records of 76 patients with knee OA (112 knees) that we had seen for US-guided injections over a defined period, we found 45 knees with no detectable effusion on static US, of which 18 (14 patients) showed fluid when scanned during voluntary quadriceps contraction. For all patients, we had recorded effusion features (physical examination, presence and size on US), and success of joint entry was determined by getting synovial fluid and/or seeing an air echo or inflow of injected material.

Results. The 14 patients we studied were obese (mean \pm SEM body mass index $32.7 \pm 2.3 \mathrm{~kg} / \mathrm{m}^{2}$; 3 morbidly obese), with moderate to severe OA by radiography in most (Kellgren/Lawrence class 3 or 4 in 10 of 14 knees for which radiographs were available). The suprapatellar synovial space seen by US was small (mean \pm SEM depth $0.38 \pm 0.04 \mathrm{~cm}$ ). Arthrocentesis obtained $0.5-16 \mathrm{ml}$ of synovial fluid (mean \pm SEM $2.9 \pm 0.6 \mathrm{ml}$ ), which correlated with the depth of effusion as seen on US with the quadriceps in maximum contraction (Spearman's $\rho=0.5597, P=0.0157$ ). In 4 knees where arthrocentesis failed to retrieve fluid, we observed at injection the inflow of material and a linear air echo.

Conclusion. US of the knee during voluntary quadriceps contraction can find effusions not detectable on static US. Such effusions provide targets for accurate aspiration and injection that would not be appreciated with static US.

\begin{abstract}
Introduction
Using ultrasound (US) to assist arthrocentesis and injection of the knee accurately obtains fluid and places injected materials more precisely than can be achieved using techniques guided only by external anatomic landmarks (1). Accuracy depends largely on US detection of intraarticular fluid, often missed by physical examination (2), which provides a "target" for the US-guided needle. USguided knee entry into the suprapatellar pouch should generally be less painful than entry via palpation-guided methods because the latter are more likely to hit bone while aiming for a reliable entry site into the joint, such as between the femur and the patella, which are landmarks that have been our habit to use. In knees where US cannot
\end{abstract}

Dr. Somers's work was supported by an NIH Clinical and Translational Science Award (grant UL1-RR024986).

${ }^{1}$ Robert W. Ike, MD, Emily C. Somers, PhD: University of Michigan, Ann Arbor; ${ }^{2}$ Erin L. Arnold, MD, William J. Arnold, MD: Illinois Bone and Joint Institute, Morton Grove.

Address correspondence to Robert W. Ike, MD, Rheumatology Division, University of Michigan Medical Center, 3918 Taubman Center, 1500 East Medical Center Drive, Ann Arbor, MI 48109-0358. E-mail: rike@umich.edu.

Submitted for publication June 23, 2009; accepted in revised form January 4, 2010. detect an effusion, guidance of joint penetration reverts to palpation of anatomic landmarks. A US-guided approach to the medial patellofemoral facet has been described for use in knees lacking an effusion (3), but still runs the risk of hitting bone during needle entry. Although methods have been described in which US detection of injected air or other contrasts can assure that a joint lacking an effusion has been entered $(4,5)$, such techniques still carry the disadvantages of palpation-guided injections.

One author (WJA) attempting to enhance an effusion in a manner similar to what can be accomplished with manual pressure on the gutters and posterior capsule asked a patient to contract her quadriceps, and saw that the effusion image on US enlarged. We have subsequently sought to elicit this phenomenon in patients undergoing USguided knee injection by asking the patient to contract their quadriceps while we continued to scan the suprapatellar region. We have found that this technique frequently shows an effusion where we could detect none previously, allowing us to obtain fluid and inject therapeutic material under direct guidance rather than having to resort to palpation-guided methods. To demonstrate the utility of this simple maneuver, we describe herein the patients we saw over a defined period of time in which this technique proved helpful. 


\section{Patients and Methods}

Patient population. We saw patients in a private practice setting (ELA, WJA) or in a clinical academic practice (RWI). All of the patients had knee osteoarthritis (OA) and pain that had not responded to more conservative medial management, consisting of analgesics, nonsteroidal antiinflammatory drugs, exercise, and joint protection. Combining experiences from a 6-month interval (RWI) and 9 separate clinic days (ELA, WJA), we saw 76 patients for USguided injection treatment. For all of the patients, we had recorded anthropomorphics, physical examination evidence for effusion, Kellgren/Lawrence (K/L) stage of knee radiographs (when available), presence and size of effusion on US, whether quadriceps contraction was required to demonstrate effusion, and the success of joint entry determined by getting synovial fluid and/or seeing an air echo or inflow of injected material. Of the 112 knees of these patients, 45 did not show an effusion on static US examination. With the patients voluntarily contracting their quadriceps, we detected suprapatellar fluid in 18 knees of 14 patients. We chose these 14 patients for the focus of this retrospective descriptive study. The protocol for retrospective data review was approved by the University of Michigan Medical School Institutional Review Board.

Qualifications of ultrasonographers. Each author performing US in this study is self-taught, with accumulated experience (at time of initiation of the study) as follows: RWI, 25 years of practice, 4 years performing US, $\sim 370$ scans; ELA, 6 years of practice, 8 years performing US, $\sim 10,000$ scans; and WJA, 37 years of practice, 6 years performing US, $\sim 10,000$ scans.

Techniques for US scanning and arthrocentesis. All of the patients gave verbal informed consent for US-guided arthrocentesis and injection. We entered joints with US guidance as has previously been described (6). We used either an ATL HDI 4000 or a GE LOGIQ e machine to perform US examinations, utilizing L12-5 38 probes or 12L-RS probes with standard musculoskeletal presets on each machine. We scanned each knee according to the European League Against Rheumatism guidelines, although we did not scan the knee in maximal flexion (a maneuver directed at the femoral articular cartilage) nor did we routinely scan the posterior region (7). We first surveyed the joint to find any evidence of a joint effusion, and then located in the longitudinal plane the largest collection of fluid. When we could not detect any fluid, we asked the patient to contract their quadriceps muscle, noting any fluid that then appeared. By the time of this study, we had incorporated this maneuver into our standard scanning protocol. We asked patients who could not contract their quadriceps on command to dorsiflex and hold against resistance their extensor hallucis longus and tibialis anterior muscles, an action that was then accompanied by quadriceps contraction. With the transducer in place, we marked an optimal site for joint entry. We cleansed the skin, and then used a sterile compound as an acoustic coupler (povidone-iodine gel [ELA, WJA] or sterile lubri- cating jelly [RWI]). After injection of a local anesthetic, we penetrated the joint while visualizing the needle with US. We removed synovial fluid until the suprapatellar cavity collapsed, and then delivered the material (corticosteroids, hyaluronate, or platelet-rich plasma according to judgment of the treating clinician), visualizing its entry into the space. In some instances, a sharp linear echo produced by the small amount of air contained in the syringe with therapeutic material provided further assurance of proper joint entry (4). We advised patients to limit their activities for the next 24-48 hours.

Statistical analysis. We computed summary statistics (means, SEMs) and examined data distributions. We used Spearman's rank correlation coefficient to assess the magnitude and significance of the association between effusion dimension and synovial fluid volume, since the latter variable was not normally distributed. We used Stata software, version 10 (Stata Corporation), to analyze the data.

\section{Results}

Characteristics of the 14 patients we studied are listed in Table 1 . There were 13 women and 1 man, ages 44-88 years (mean \pm SEM age $64.7 \pm 2.9$ years). Most were obese (body mass index range $20.1-49.9 \mathrm{~kg} / \mathrm{m}^{2}$, mean $\pm \mathrm{SEM}$ $32.7 \pm 2.3 \mathrm{~kg} / \mathrm{m}^{2}$ ), with 3 classifiable as morbidly obese $\left(\mathrm{BMI}>40 \mathrm{~kg} / \mathrm{m}^{2}\right)$. OA stage as determined by radiography tended toward more severe, with (K/L) stage 3 or 4 seen in 10 of the 14 knees for which radiographs were available. Physical examination suggested the presence of an effusion in only 1 knee (positive bulge sign).

The suprapatellar synovial space detected by US was small (mean \pm SEM maximum depth $0.38 \pm 0.04 \mathrm{~cm}$ ) (Figure 1). The volume of fluid obtained at arthrocentesis ranged from 0.5 to $16 \mathrm{ml}$ (mean \pm SEM $2.9 \pm 0.6 \mathrm{~m} / \mathrm{n}$ ) and correlated significantly with depth of effusion as seen on US with the quadriceps in maximum contraction (Figure 2). We did not seek to evacuate all synovial fluid in every case, because in some instances contraction of the synovial cavity with aspiration threatened to reduce the space beyond the reach of the needle tip. In 4 cases, we could not obtain synovial fluid despite visualizing the needle tip within the synovial space. In all of these cases, we observed injected material flow into the space and also saw a linear air echo (Figure 3). No patient reported immediate complications of the procedure.

\section{Discussion}

We have described a simple technique that can detect synovial fluid in knees for which static US had failed to find evidence for an effusion. The phenomenon of suprapatellar bursal fluid appearing on US with quadriceps contraction was described many years ago by Martino et al (8), who recognized the suprapatellar bursa in $16 \%$ of 50 normal knees they examined, and then found suprapatellar hypoechogenicity in an additional $30 \%$ of knees on quadriceps contraction. However, mention of this phenomenon has not entered general writings on knee US. Detecting occult effusions with quadriceps contraction in our series permitted aspiration of synovial fluid and con- 


\begin{tabular}{|c|c|c|c|c|c|c|c|}
\hline Patient & $\begin{array}{c}\text { Age, } \\
\text { years/sex }\end{array}$ & $\begin{array}{l}\text { BMI, } \\
\mathrm{kg} / \mathrm{m}^{2}\end{array}$ & Knee & $\begin{array}{l}\text { Radiograph grade } \\
\text { (Kellgren/Lawrence) }\end{array}$ & $\begin{array}{c}\text { Physical } \\
\text { examination }\end{array}$ & $\begin{array}{l}\text { Maximum depth } \\
\text { of effusion, cm }\end{array}$ & $\begin{array}{c}\text { Volume of } \\
\text { synovial fluid } \\
\text { obtained, ml }\end{array}$ \\
\hline 1 & $44 / F$ & 49.9 & Left & 3 & - & 0.3 & 3.0 \\
\hline \multirow[t]{2}{*}{2} & $60 / F$ & 42.0 & Right & 4 & - & 0.2 & 0.1 \\
\hline & & & Left & 4 & - & 0.6 & 1.0 \\
\hline \multirow[t]{2}{*}{3} & $72 / \mathrm{F}$ & 45.4 & Right & 4 & - & 0.3 & 2.0 \\
\hline & & & Left & 4 & - & 0.3 & 0.5 \\
\hline \multirow[t]{2}{*}{4} & $71 / \mathrm{F}$ & 25.2 & Right & 3 & - & 0.4 & 6.0 \\
\hline & & & Left & 2 & - & 0.4 & 4.0 \\
\hline 5 & $65 / F$ & 24.6 & Right & $\mathrm{n} / \mathrm{a}$ & - & 0.2 & 5.0 \\
\hline 6 & $87 / F$ & 28.5 & Left & 2 & - & 0.3 & 1.0 \\
\hline 7 & $75 / F$ & 38.8 & Left & $\mathrm{n} / \mathrm{a}$ & - & 0.6 & 2.0 \\
\hline 8 & $79 / F$ & 24.9 & Right & 4 & - & 0.2 & $0+$ \\
\hline \multirow[t]{2}{*}{9} & $59 / \mathrm{F}$ & 34.6 & Right & 2 & - & 0.2 & $0+$ \\
\hline & & & Left & 3 & - & 0.2 & $0+$ \\
\hline 10 & $68 / F$ & 32.9 & Left & $\mathrm{n} / \mathrm{a}$ & + & 0.6 & 16 \\
\hline 11 & $88 / F$ & 20.1 & Right & 4 & - & 0.4 & 3.0 \\
\hline 12 & $67 / F$ & 30.4 & Left & $\mathrm{n} / \mathrm{a}$ & - & 0.7 & 6.0 \\
\hline 13 & $60 / \mathrm{M}$ & 30.3 & Left & 3 & - & 0.5 & 0.5 \\
\hline 14 & $49 / F$ & 30.0 & Left & 1 & - & 0.5 & 2.0 \\
\hline
\end{tabular}

firmed intraarticular placement of injected material. These successes came in the patients who often presented challenges of obesity and advanced arthritis. Such cases comprise an ever-growing number of our patients as the general population ages and becomes more obese.

Our study has certain weaknesses. This was a retrospective study examining a small number of patients. We collected patients in different manners at each site, looking at all of the patients over a defined period of time at an academic site, while collecting data from patients seen only on certain defined days at the private practice site. Our goal was to accumulate a sufficient number of cases to provide a reasonable illustration of this phenomenon, which we managed to do by taking samples from the private practice environment to be added to the academic set. The true prevalence of the quadriceps contraction phenomenon in knee OA will be better delineated by prospective studies. The relationship we demonstrated between the size of effusion on US and synovial fluid volume

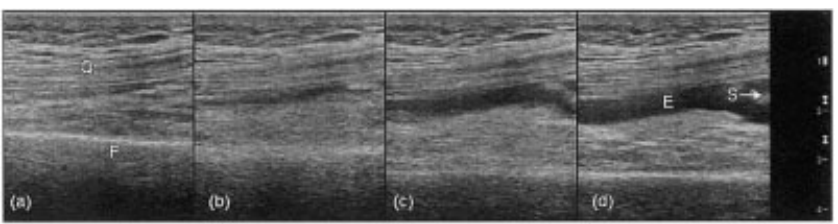

Figure 1. Sequence of panels depicting the appearance of effusions with quadriceps contraction. A, Sagittal (longitudinal) scan at rest. No hypoechoic/anechoic material can be seen to suggest effusion between the quadriceps tendon (Q) and femur (F). B, Initiation of contraction. Note the slender strip of hypoechoic material appearing. C, Midcontraction with hypoechoic effusion evident. D, Maximum contraction. Effusion (E) of maximum depth of $0.7 \mathrm{~cm}$. Note the frond of the synovium (S). Digits in the black strip to the right of the panels indicate the centimeters of depth. obtained makes intuitive sense, but may not be as strong as our data indicated. We were more cautious evacuating small effusions out of concern for collapsing the space and pulling boundaries of the capsule away from the needle tip, which could lead to an extraarticular placement. Therefore, the small volumes obtained from smaller spaces might underrepresent true intraarticular volumes, whereas larger volumes from apparently larger effusions could reflect in part the confidence with which we might attempt to empty those effusions.

Studies analyzing pressure-volume relationships in normal and arthritic joints have interpreted findings mainly because they might contribute to the pathogenesis

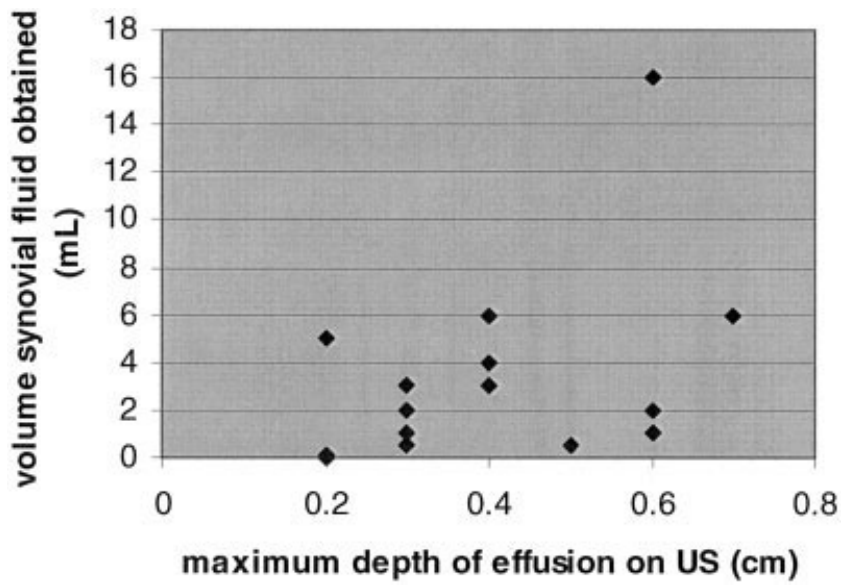

Figure 2. Relationship of the volume of synovial fluid obtained to the maximum depth of suprapatellar effusion seen on ultrasound (US). Note that not all of the effusions were completely evacuated. The relationship is statistically significant (Spearman's $\rho=$ $0.5597, P=0.0157$ ). 


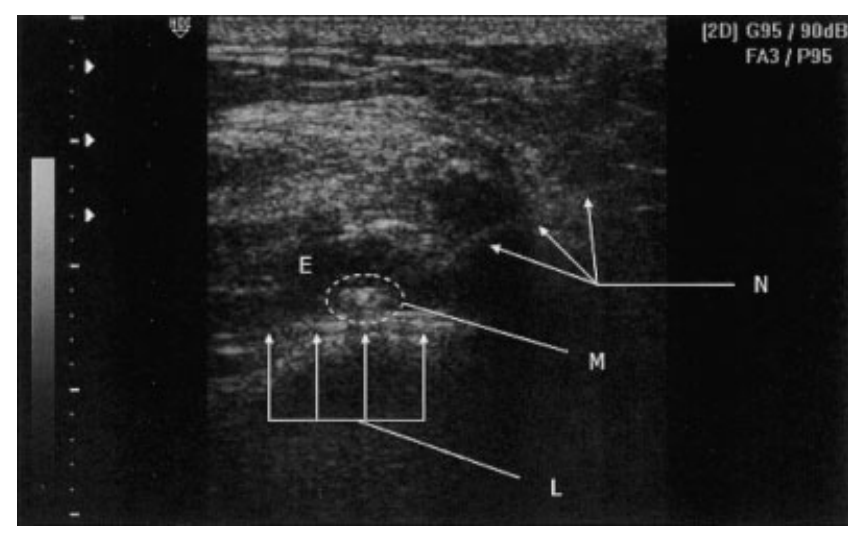

Figure 3. Depiction of successful intraarticular placement of hyaluronate (suprapatellar transverse view). The needle $(\mathbf{N})$ penetrates the small effusion (E), placing a globule of therapeutic material (M; here hyaluronate) with generation of linear air echo (L).

both of symptoms and of joint damage arising from changes in synovial perfusion, supporting structures and periarticular musculature $(9,10)$. However, some observed phenomena are applicable to clinical practice, particularly arthrocentesis. Joints accommodate their largest volume when intraarticular pressure is least, which for the knee occurs at a range of 15-60 degrees in knees with existing effusions (11); joint capsule compliance, i.e., the change in pressure produced by an increase in volume, is greatest in a range of 0-30 degrees, diminishing with greater flexion (12). Increased intraarticular pressure forces any synovial fluid to areas of greatest compliance (13), which for the knee are the suprapatellar recess and peripatellar gutters. Quadriceps contraction substantially increases intraarticular pressure (14), which would increase the amount of fluid in these compliant regions. Therefore, we generally position patients for arthrocentesis with the knee extended or in slight flexion, but can produce larger amounts of suprapatellar fluid by having the patient contract their quadriceps.

It is well established that US detects many knee effusions missed by physical examination (2) and improves the accuracy of knee arthrocentesis (1). However, it remains controversial whether the improved accuracy of US-guided injections translates into a better clinical outcome (15). Such a relationship has been shown by prospective controlled trials for the shoulder (16), and for the short term (2 weeks) in a number of different joints (17); however, another recent study could find no difference in outcome for a number of different joints with rheumatoid arthritis (RA) in which injection was performed with guidance either by palpation or US (18). An older prospective study found superior outcomes following corticosteroid injections to knees with RA in the group where joint drainage preceded injection (19); at least some of this difference could be interpreted as being due to the greater chance that knees first drained were more likely to have been successfully entered. Assuring accurate intraarticular placement may be more important for larger molecular weight biologic compounds (e.g., hyaluronate, cytokines and their modulators, growth factors) because their actions require direct contact with the synovium and/or cartilage and they would not likely diffuse into a joint from extraarticular placement.

Therefore, US has an important role in guiding needles into joints. Obtaining synovial fluid for analysis is easier if the arthrocentesis needle is localized to a fluid containing space. When US of the knee does not detect a synovial space, repeat examination with the patient voluntarily contracting the quadriceps muscle can often find fluid, and thus be amenable to an accurate aspiration and injection.

\section{AUTHOR CONTRIBUTIONS}

All authors were involved in drafting the article or revising it critically for important intellectual content, and all authors approved the final version to be published. Dr. Ike had full access to all of the data in the study and takes responsibility for the integrity of the data and the accuracy of the data analysis.

Study conception and design. Ike, William J. Arnold.

Acquisition of data. Ike, Erin L. Arnold, William J. Arnold. Analysis and interpretation of data. Ike, Somers.

\section{REFERENCES}

1. Balint PV, Kane D, Hunter J, McInnes IB, Field M, Sturrock RD. Ultrasound guided versus conventional joint and soft tissue aspiration in rheumatology practice: a pilot study. J Rheumatol 2002;29:2209-13.

2. Kane D, Balint PV, Sturrock RD. Ultrasonography is superior to clinical examination in the detection and localization of knee joint effusion in rheumatoid arthritis. J Rheumatol 2003; 30:966-71.

3. Luck LJ. Musculoskeletal ultrasound intervention: principles and advances. Radiol Clin North Am 2008;46:515-33.

4. Qvistgaard E, Kristoffersen H, Terslev L, Danneskiold-Samsoe B, Torp-Pedersen S, Bliddal H. Guidance by ultrasound of intra-articular injections in the knee and hip joints. Osteoarthritis Cartilage 2001;9:512-7.

5. Koski JM, Hermunen HS, Kilponen VM, Saarakkala SJ, Hakulinen UK, Heikkinen JO. Verification of palpation-guided intra-articular injections using glucocorticoid-air-saline mixture and ultrasound imaging (GAS-graphy). Clin Exp Rheumatol 2006;24:247-52.

6. Grassi W, Farina A, Filippucci E, Cervini C. Sonographically guided procedures in rheumatology. Semin Arthritis Rheum 2001;30:347-53.

7. Backhaus M, Burmeister GR, Gerber T, Grassi W, Machold KP, Swen WA, et al. Guidelines for musculoskeletal ultrasound in rheumatology. Ann Rheum Dis 2001;60:641-9.

8. Martino F, Angelelli G, Ettorre GC, Macarini L, Patella V, Moretti $\mathrm{B}$, et al. The normal aspect of the suprapatellar bursa in echography of the knee. Radiol Med 1992;83:43-8. In Italian.

9. Levick JR. Joint pressure-volume studies: their importance, design, and interpretation. J Rheumatol 1983;10:353-7.

10. Simkin PA. Feeling the pressure. Ann Rheum Dis 1995;54: 611-2.

11. Eyring EJ, Murray WR. The effect of joint position on the pressure of intra-articular effusion. J Bone Joint Surg Am 1964;46:1235-41.

12. Funk DA, Noyes FR, Grood ES, Hoffman SD. Effect of flexion angle on the pressure-volume of the human knee. Arthroscopy 1991;7:86-90.

13. Myers DB, Palmer DG. Capsular compliance and pressurevolume relationships in normal and arthritic knees. J Bone Joint Surg Br 1972;54:710-6. 
14. Deandrade JR, Grant C, Dixon AS. Joint distension and reflex muscle inhibition in the knee. J Bone Jt Surg Am 1965;47: 313-22.

15. Hall S, Buchbinder R. Do imaging methods that guide needle placement improve outcome? Ann Rheum Dis 2004;63: 1007-8.

16. Naredo E, Cabero F, Beneyto P, Cruz A, Mondejar B, Uson J, et al. A randomized comparative study of short term response to injection versus sonographic-guided injection of local corticosteroids in patients with painful shoulder. J Rheumatol 2004;31:308-14.
17. Sibbitt WL Jr, Peisajovich A, Michael AA, Park KS, Sibbitt RR, Band PA, et al. Does sonographic needle guidance affect the clinical outcome of intraarticular injections? J Rheumatol 2009;36:1892-902.

18. Cunnington J, Marshall N, Hide G, Platt P, Kane D. A randomized controlled trial of ultrasound versus clinical guided intra-articular corticosteroid injection in inflammatory arthritis [abstract]. Arthritis Rheum 2008;58 Suppl:S639.

19. Weitoft T, Uddenfeldt P. Importance of synovial fluid aspiration when injecting intra-articular corticosteroids. Ann Rheum Dis 2000;59:233-5. 